\title{
Autoradiographische Darstellung von Phospho-Proteinen in Gewebsschnitten mit Hilfe von Radiophosphor*
}

\author{
Von Eberhard Harbers und Rupert BackmanN \\ Aus dem Institut für medizinische Physik und Biophysik der Universität Göttingen \\ (Direktor Prof. Dr. E. Witte) \\ (Z. Naturforschg. 10 b, 385-388 [1955]; eingegangen am 11. Mai 1955)
}

\begin{abstract}
Bei der autoradiographischen Lokalisation von Radiophosphor in Gewebsschnitten geht ein beträchtlicher Teil der in der Photoemulsion ausgelösten Schwärzung auf radioaktiv gewordene Phospho-Proteine zurück. Die Übertragung der Gewebsfraktionierung nach S c h n e i d e r auf histologische Schnitte macht es möglich, bei der Autoradiographie die Phospho-Proteine angenähert selektiv darzustellen. Es wurde der Anteil der Phospho-Proteine an der gesamten Radioaktivität des Gewebes nach der im einzelnen beschriebenen Vorbehandlung der Schnitte quantitativ bestimmt. Vergleichende Untersuchungen an fixierten Organen und am Gewebshomogenat ergaben, daß die Phospho-Proteine bei Fixierung in Carnoyscher Lösung durch die histologische Vorbehandlung keine Verluste erleiden.
\end{abstract}

$\mathrm{R}$ adioaktiver Phosphor, als anorganisches Phosphat verabfolgt, wird von tierischen Organen in beträchtlicher Menge in die sog. Phospho-Proteine eingebaut. Da sich diese aus Gewebsschnitten praktisch kaum extrahieren lassen ${ }^{1}$, ist bei der autoradiographischen Untersuchung solcher Organe die in der Photoemulsion ausgelöste Schwärzung zum großen Teil auf radioaktiv gewordene Phospho-Proteine zurückzuführen. Der den Phospho-Proteinen zugehörige Anteil am Gesamtgehalt des Gewebes an Radiophosphor hängt von verschiedenen Faktoren ab, wie Organart, Zeitspanne zwischen Verabfolgung des Isotops und Tötung der Versuchstiere, Vorbehandlung des Gewebes, und so weiter. Um eine bessere Bewertung der autoradiographischen Präparate entsprechend den Forderungen der Histochemie zu ermöglichen ${ }^{2}$, ist es wünschenswert, die Phospho-Proteine möglichst selektiv darzustellen. Hierzu sind die Schnitte vor dem Zusammenbringen mit der Photoemulsion so zu behandeln, daß alle phosphor-haltigen Gewebsbestandteile bis auf die Phospho-Proteine extrahiert werden. Zu diesem Zweck wurde das Fraktionierungsverfahren von $\mathrm{S} \mathrm{chneide}{ }^{3}$, das bereits als histochemische Methode zur Extraktion der $\mathrm{Nu}-$ cleinsäuren aus Gewebsschnitten benutzt wird, angewandt.

* Die Untersuchungen wurden mit Mitteln der De u t schen Forschungsgemeins chaf $t$ durchgeführt.

1 E. $\mathrm{H}$ a r b e r s u. K. N e u m a n n, Z. Naturforschg. 9 b, 175 [1954].

2 E. H a rbers u. K. N e u m a n n, Klin. Wschr. 32, 337 [1954].

3 W. C. S c hneider, J. biol. Chemistry 161, 293 [1945].
In den meisten Organen ist die Menge der Phospho-Proteine recht klein. Ihre Bezeichnung gründet sich darauf, daß bei alkalischer Hydrolyse der nichtsäurelöslichen Phosphat-Fraktion aus Eiweiß-Stoffen anorganisches Phosphat freigesetzt wird. Über Art des Einbaues dieses Phosphats in die Proteine, ihre funktionelle Bedeutung usw. ist bisher noch wenig bekannt. Nach neueren Untersuchungen von Ke nne dy ${ }^{4,5}$ liegt das Phosphat in erster Linie als Phospho-Serin vor. Davidson ${ }^{6}$ machte darauf aufmerksam, daß die Phospho-Proteine ungewöhnlich viel Radiophosphor aufnehmen, also offenbar einen sehr intensiven Umsatz haben. Zunächst gehegte Zweifel, ob der hohe Gehalt an ${ }^{32} \mathrm{P}$ tatsächlich nur den Phospho-Proteinen zuzuschreiben sei und nicht von Verunreinigungen herrühre, wurden durch weitere Untersuchungen, insbesondere von $\mathrm{J}$ ohn s on und Albert ${ }^{7}$, beseitigt. Die Befunde der letztgenannten Autoren weisen zugleich auf die Bedeutung der Phospho-Proteine für den Zellstoffwechsel hin.

\section{Methodik}

Versuchstiere waren Wistar-Ratten und erbreine Mäuse der Stämme B 1 und C $3 \mathrm{H}$. Untersucht wurde in erster Linie die Leber. Abgewogene Gewebsmengen wurden parallel einmal als Gewebshomogenat aufgearbeitet, zum anderen in Carnoyscher Lösung fixiert und eingebettet.

4 E. P. K e nne d y, Proc. Amer. Assoc. Cancer Res. 1, 29 [1953].

5 E. P. Kennedy u. S. W. S mith, J. biol. Chemistry 207, 153 [1954].

6 J. N. D a vid s on, S. C. Frazer u. W. C. H u t c. his on, Biochem. J. 49, 311 [1951].

7 R. M. J o h n s o n u. S. A l b e r t, J. biol. Chemistry 200, 335 [1953]. 
Die Gewebsblöcke wurden mit dem Mikrotom völlig aufgeschnitten und die gesamten Schnitte gemeinsam den histochemischen Reaktionen ausgesetzt. Zur quantitativen analytischen Auswertung (Phosphatbestimmung und Zählrohrmessung) wurden die Schnitte entweder verascht oder noch weiter fraktioniert, wie nachstehend beschrieben. Die Injektion des Radiophosphor erfolgte intraperitoneal (als $\mathrm{Na}_{2} \mathrm{HPO}_{4}$ ); Dosis $2 \mu \mathrm{C} / \mathrm{g}$ Körpergewicht für die chemischanalytischen, $10-20 \mu \mathrm{C} / \mathrm{g}$ für die autoradiographischen Untersuchungen.

a) Untersuchungen am Gewebshomoge$\mathrm{nat}$ : Aus frisch abgewogenem Gewebe (200-500 mg) wurden nach Homogenisieren zunächst mit eisgekühlter 10-proz. Trichloressigsäure die säurelösliche Phosphatfraktion, anschließend mit Alkohol und Alkohol-Äther die Phospholipoide extrahiert. Diese Fraktionierung erfolgte mittels der Frittenmethode von Lavik ${ }^{8}$. Der Gewebsrückstand wurde nun zur Extraktion der Nucleinsäuren 15 min lang mit 5-proz. Trichloressigsäure bei $90^{\circ} \mathrm{C}$ im Wasserbad exponiert. Der dann noch im Gewebe verbliebene Phosphor soll den Phospho-Proteinen zuzuordnen sein. Vergleichende Untersuchungen verschiedener Autoren zeigten jedoch, daß die auf diese Weise ermittelten Analysewerte immer größer waren als die, welche sich bei Anwendung der Methode von $\mathrm{S} \mathrm{chmidt}$ und Th a $n \mathrm{nh}$ a u s e ${ }^{\mathbf{9}}$ ergaben. J ohns on und A l ber $\mathrm{t}^{\mathbf{7}}$ konnten nun zeigen, daß die Extraktion durch die 5-proz. Trichloressigsäure offenbar unvollständig ist. Wenn dieser jedoch eine alkalische Hydrolyse mit $n-\mathrm{NaOH}$ (20 Stdn. bei $37^{\circ} \mathrm{C}$ ) angeschlossen und das dabei freigesetzte anorganische Phosphat bestimmt wurde, entsprachen die Analysewerte denen der S c h midt - Than $\mathrm{nh}$ a u s e rMethode. Aus diesem Grunde wurde von uns ebenfalls im Anschluß an die Extraktion der Nucleinsäuren der Gewebsrückstand mit $n-\mathrm{NaOH} 18$ bis $20 \mathrm{Stdn}$. bei $37^{\circ} \mathrm{C}$ exponiert. Aus dem nach Neutralisieren und Fällen mit 5-proz. Trichloressigsäure nun gewonnenen Extrakt wurde das freie Orthophosphat entweder mit Magnesia-Mixtur gefällt oder zur Zählrohrmessung papierchromatographisch isoliert 10. Die Phosphatbestimmung erfolgte kolorimetrisch nach den Angaben von Go mori ${ }^{11}$. Es wurden jeweils wenigstens 3 Parallelanalysen durchgeführt. Der mittlere Fehler der Einzelwerte lag um $\pm 10 \%$, stieg bei der nachstehend beschriebenen quantitativen Untersuchung der histochemischen Reaktionen mitunter bis auf 20 Prozent.

b) Quantitative Untersuchung der histochemis chen Reaktionen: Die Fixierung des Gewebes erfolgte in Carnoyscher Lösung, da dabei schon eine weitgehende Extraktion der säurelöslichen Phosphate und der Phospholipoide stattfindet. Nach völligem Aufschneiden der Gewebsblöcke wurden alle Schnitte gemeinsam mit Xylol entparaffiniert und in üblicher Weise die absteigende Alkoholreihe bis zum dest. Wasser durchgeführt. Zur gründlicheren Extraktion der Phospholipoide wurden dabei die Schnitte entsprechend den Angaben von B rattgard und $\mathrm{Hy}$ den 12 nach dem 96-proz. Alko-

8 P. S. Lavik, H. H a r r ing ton u. G. W. Buckalo o, Western Res. Univ., Atomic Energy Med. Res. Proj., Unclassified Docum., NYO-1625 [1951].

9 G. S chmidt u. S. J. Th an n ha s e r, J. biol. Chemistry 161, 83 [1945]. hol zunächst 2-mal mit Chloroform behandelt. Nach Extraktion der Nucleinsäuren mit 5-proz. Trichloressigsäure bei $90^{\circ} \mathrm{C}(15 \mathrm{~min})$ und anschließendem mehrfachen Spülen mit 5-proz. Trichloressigsäure wurden die Gewebsschnitte entweder verascht (zur Bestimmung des Gesamtphosphats und des Radiophosphors) oder weiterfraktioniert wie bei den Untersuchungen am Gewebshomogenat.

Die praktische Durchführung dieser Untersuchungen wurde wesentlich dadurch erleichtert, daß die Paraffinschnitte jeweils in eine ausreichend große Fritte gebracht wurden, deren Boden mit einer festen Schicht Filterhilfe („Celite“ der Johns-Manville Company, Ltd., U.S.A., oder Nr. 0114 von Schleicher \& Schüll) bedeckt war. Durch vorsichtiges Absaugen des Xylols, Alkohols usw. konnten so die verschiedenen bei der histologischen Behandlung des Gewebes üblichen Schritte durchgeführt werden, ohne daß durch ständiges Überführen Verluste eintraten. Die Filterhilfe am Boden der Fritten darf hierbei zunächst nicht aufgerührt werden; vielmehr bildet sie eine Art Schlammfang und verhindert damit ein Verstopfen der Fritte. Im Anschluß an die histochemischen Reaktionen kann dann in der gleichen Fritte die weitere Fraktionierung vorgenommen werden.

c) A u toradiographis che Technik: Die Behandlung der Schnitte erfolgte in der gleichen Weise, wie im vorigen Abschnitt beschrieben. Nach Extraktion der Nucleinsäuren wurden die Objektträger mit den Gewebsschnitten noch je 3-mal in 5-proz. Trichloressigsäure (Zimmertemperatur) gebracht und nach 3-maligem Wässern (je 5 min) mit Hämalaun-Eosin schwach gefärbt. Durch die Trichloressigsäure-Extraktion verlor das Gewebe fast völlig seine Basophilie. Zur Autoradiographie wurde Stripping-Film (Kodak/England) nach der Methode von Pelc ${ }^{13}$ verwendet. Gemäß den Vorschriften von Kodak wurden die Objektträger vor der Benutzung mit verdünnter Chromschwefelsäure gereinigt, nach gründlichem Wässern kurz in eine 0,5-proz. Gelatinelösung getaucht und an der Luft getrocknet; auf diese Weise kam es zu einer zuverlässigen Bindung des anschließend in der Dunkelkammer aufgebrachten Films. Entwickelt wurde nach beendeter Exposition mit Kodak D-19b-Entwickler (4 bis $5 \mathrm{~min}$ ), fixiert in 30-proz. Natriumthiosulfatlösung ( $30 \mathrm{~min})$; anschließend wurden die Präparate gut gewässert und dann getrocknet.

\section{Ergebnisse}

Es wurde zunächst die Extraktion, wie sie Schneid e $r^{3}$ ursprünglich beschrieb, parallel am Homogenat und an Carnoy-fixierten Geweben durchgeführt. Dabei ergab sich regelmäßig, daß die Menge des im Gewebe verbliebenen Gesamt-Phosphors unter dem Einfluß der histologischen Behandlung wesentlich kleiner wurde. Die spezifische Aktivität dieses Phosphats

10 R. B a ckmann u. E. Harbers, Naturwissenschaften 41, 64 [1954].

11 G. Go m or i, J. Lab. clin. Med. 27, 955 [1941].

12 S. O. B r attgard u. H. H y d e n, Acta Radiologica [Stockholm] Suppl. 94, [1952].

13 S. R. P e l c, Nature [London] 160, 749 [1947]. 


\begin{tabular}{|c|c|c|c|c|}
\hline \multirow{2}{*}{ Gewebsart } & \multicolumn{2}{|c|}{ Gewebshomogenat } & \multicolumn{2}{|c|}{$\begin{array}{c}\text { Gewebsschnitte, } \\
\text { fixiert }\end{array}$} \\
\cline { 2 - 5 } & $\begin{array}{r}\text { mg P/g } \\
\text { Gewebe }\end{array}$ & $\begin{array}{r}\text { spezif. } \\
\text { Aktiv. }\end{array}$ & $\begin{array}{c}\text { mg P/g } \\
\text { Gewebe }\end{array}$ & $\begin{array}{c}\text { spezif. } \\
\text { Aktiv. }\end{array}$ \\
\hline $\begin{array}{c}\text { Mäuseleber } \\
\text { Rattenleber }\end{array}$ & 0,35 & 147000 & 0,112 & 402000 \\
0,39 & 83000 & 0,149 & 135000 \\
\hline
\end{tabular}

Tab. 1. Vergleich der Analysewerte für Phospho-Proteine von Gewebshomogenat und fixiertem Gewebe bei Anwendung der Methode von S chneider ${ }^{3}$. Die Zahlen sind jeweils Mittelwerte von 3 Parallelanalysen. Die spezifische Aktivität ist angegeben in Zählrohrimpulsen/min/mg $\mathrm{P}$.

\begin{tabular}{|l|r|}
\hline & Aktivität/g Gewebe (Rattenleber) \\
\hline $\begin{array}{l}\text { Gewebshomogenat } \\
\text { Carnoy fixiert }\end{array}$ & $14200 \pm 1380$ \\
$15200 \pm 1710$ \\
\hline
\end{tabular}

Tab. 2. Vergleich der Aktivitäten des papierchromatographisch isolierten anorganischen Phosphats, das im Anschluß an die Fraktionierung nach Schneider durch alkalische Hydrolyse freigesetzt wurde. Die Aktivität ist angegeben in Zählrohrimpulsen/min.

war dagegen im fixierten Gewebe immer sehr viel größer als im Homogenat (Beispiel in Tab. 1).

Nun sind erfahrungsgemäß die Phospho-Proteine unter den gewählten Versuchsbedingungen (die Zeitspanne zwischen Verabfolgung des Radiophosphors und der Tötung der Versuchstiere betrug jeweils nur wenige Stdn.) stets die Phosphat-Fraktion mit der höchsten spezifischen Aktivität. So war aus diesen Befunden zu schließen, daß durch die Fixierung des $\mathrm{Ge}$ webes kaum Phospho-Proteine extrahiert wurden, wohl aber ein Teil der Substanzen, welche bei der $\mathrm{S}$ chneider-Methode am Gewebshomogenat zu zu hohen Analysewerten führt ${ }^{7}$. Um die Richtigkeit dieser Überlegung zu prüfen, wurde nach Extraktion der Nucleinsäuren mit heißer 5-proz. Trichloressigsäure der Gewebsrückstand mit $n$ - $\mathrm{NaOH}$ hydrolysiert, um den Phosphor der Phospho-Proteine als anorganisches Phosphat freizusetzen. Nach papierchromatographischer Isolierung dieses Phosphats ergaben Zählrohrmessungen, daß die Aktivität pro g Gewebe in Homogenat und fixiertem Gewebe praktisch gleich waren (Tab. 2). Damit wird die Deutung der zuerst geschilderten Befunde, daß durch Fixierung keine Phospho-Proteine verlorengehen, als richtig bestätigt. Die Isolierung des anorganischen Phosphats aus dem alkalischen Hydrolysat erlaubt jedoch zugleich, fest-

\begin{tabular}{|c|c|c|c|}
\hline \multirow[b]{2}{*}{ Gewebsart } & \multicolumn{2}{|c|}{ Aktivität/g Gewebe } & \multirow{2}{*}{$\begin{array}{c}\text { Anteil der } \\
\text { Phospho-Pro- } \\
\text { teine an der } \\
\text { verbliebenen } \\
\text { gesamten } \\
\text { Radioaktivität } \\
\text { des Gewebes } \\
{[\%]}\end{array}$} \\
\hline & $\begin{array}{l}\text { nach Vor- } \\
\text { behandlung } \\
\text { entspr. der } \\
\text { Methode von } \\
\text { Schneider }\end{array}$ & $\begin{array}{l}\text { Phospho-Pro- } \\
\text { teine (anorg. } \\
\text { Phosphat } \\
\text { nach alkal. } \\
\text { Hydrolyse) }\end{array}$ & \\
\hline Leber & 19900 & 15250 & 77 \\
\hline Niere & 17900 & 15100 & 84 \\
\hline Gehirn & 860 & 600 & 70 \\
\hline Milz & 36800 & 23900 & 65 \\
\hline $\begin{array}{l}\text { Tumor } \\
\text { (Mammacar- } \\
\text { cinom } \mathrm{C}_{3} \mathrm{H} \text { ) }\end{array}$ & 15700 & 12400 & 79 \\
\hline
\end{tabular}

Tab. 3. Bestimmung des Anteils der Phospho-Proteine an der gesamten verbliebenen Radioaktivität von Gewebsschnitten, nachdem diese entsprechend der Methode von $\mathrm{S}$ chneider vorbehandelt waren. Zeitspanne zwischen Verabfolgung des Radiophosphors und der Tötung der Versuchstiere (C 3 H-Mäuse) jeweils 3 Stunden. Die Aktivität ist angegeben in Zählrohrimpulsen/min.

zustellen, welchen Anteil die Phospho-Proteine an der gesamten Radioaktivität der Gewebsschnitte haben, wenn zuvor die einfache Fraktionierung nach Schneider durchgeführt wurde. Je größer dieser Anteil, um so mehr ist die Autoradiographie derartig vorbehandelter Schnitte als selektive der PhosphoProteine anzusprechen. Tab. 3 gibt die Ergebnisse solcher Analysen von verschiedenen Geweben wieder und läßt erkennen, daß in der Tat der überwiegende Teil der Radioaktivität den Phospho-Proteinen zuzuordnen ist.

Bei erster Anwendung der geschilderten Methode zur Untersuchung verschiedener Organe der Maus ließen sich von Leber, Milz, Niere und einem Tumor (Mäusesarcom 180) positive Autoradiographien herstellen. Die Aktivität war dabei innerhalb der Gewebe offenbar recht gleichmäßig verteilt. Von den Phospho-Proteinen des Tumors wurde anscheinend in nekrotischen Gewebsbereichen wenig oder gar kein Radio-Phosphat mehr aufgenommen. Wenn andererseits das Gewebe nicht mit Trichloressigsäure extrahiert worden war, ließ sich auch in den Nekrosen Radiophosphor nachweisen.

Da uns leider nur eine recht unempfindliche Photoemulsion zur Verfügung stand, ließen sich von anderen Organen mit geringerem Umsatz der PhosphoProteine (Gehirn, Lungen, Herz und Testes) nach der Trichloressigsäure-Extraktion keine brauchbaren autoradiographischen Präparate herstellen, es sei denn, die Dosis des verabfolgten ${ }^{32} \mathrm{P}$ wäre um ein Vielfaches 
erhöht worden. Mit empfindlicheren Emulsionen dürfte jedoch die autoradiographische Darstellung der Phospho-Proteine in den meisten Organen keine prinzipiellen Schwierigkeiten bereiten.

\section{Diskussion}

Die geschilderten Untersuchungen haben gezeigt, daß eine angenähert selektive autoradiographische Darstellung der Phospho-Proteine in tierischen Geweben mit einfachen Mitteln möglich ist; die übrigen Phosphat-Fraktionen nehmen alle relativ wenig. ${ }^{32} \mathrm{P}$ auf und lassen sich außerdem verhältnismäßig gut aus den Gewebsschnitten extrahieren. Die Situation ist damit gerade umgekehrt wie bei der autoradiographischen Darstellung der Nucleinsäuren, die ohne Überlagerung durch die Radioaktivität der PhosphoProteine nicht möglich ist ${ }^{1}$. Bei Fixierung des Gewebes in Carnoyscher Lösung kommt es zu keinen Verlusten der Phospho-Proteine; dagegen wird durch diese Gewebsbehandlung ein Teil derjenigen Phosphor-Verbindungen mit extrahiert, welche bei Anwendung der Methode von $\mathrm{Schneider^{3 }}$ am Gewebshomogenat $\mathrm{zu}$ hohe Analysewerte bewirken ${ }^{7}$. Die günstigen Verhältnisse, wie sie die Werte der Tab. 3 wiedergeben, bestehen jedoch nur für eine begrenzte Zeitspanne nach der Injektion des Radiophosphors, während der es - entsprechend dem hohen Umsatz der Phospho-Proteine - zu einem steilen Anstieg der spezifischen Aktivität kommt ${ }^{14}$. Die Zeit zwischen der Verabfolgung des radioaktiven Phosphats und der Tötung der Versuchstiere sollte mindestens 1 Stde. betragen, da sonst das anorganische Phosphat mit seinem anfangs sehr hohen Gehalt an ${ }^{32} \mathrm{P}$ nicht ausreichend aus den Gewebsschnitten extrahiert werden kann. Wenn andererseits mehr als 5 bis

14 E. H a r b e r s, Klin. Wschr. 32, 392 [1954].
6 Stdn. zwischen Injektion und Tötung verstreichen, besteht die Gefahr, daß die Phospho-Proteine bereits das Maximum ihrer ${ }^{32} \mathrm{P}$-Aufnahme überschritten haben und sich nun die unvollständige Extraktion der Nucleinsäuren, die derweil auch beträchtlich radioaktiv geworden sind, ungünstig auswirkt.

Nach Abschluß unserer Versuche wurde uns eine Arbeit von T a y l o r ${ }^{15}$ bekannt, in der u. a. über die autoradiographische Darstellung der Phospho-Proteine an pflanzlichen Objekten berichtet wird. Die Extraktion der Nucleinsäuren erfolgte dabei ebenfalls mit heißer Trichloressigsäure oder durch enzymatische Hydrolyse. Eine quantitative Bestimmung des Anteils der Phospho-Proteine an der nach dieser Vorbehandlung im Gewebe verbliebenen Radioaktivität wurde jedoch nicht vorgenommen.

Da die Phospho-Proteine allen Versuchen, sie aus Gewebsschnitten unter Erhaltung der Struktur zu extrahieren, widerstehen, hat zwangsläufig der von ihnen aufgenommene Radiophosphor einen mehr oder weniger großen Anteil an der gesamten Radioaktivität des Gewebes. Das Ausmaß dieses Anteiles wird leicht unterschätzt, und so werden daher häufig die Phospho-Proteine - zwar nicht selektiv autoradiographisch dargestellt, wenn durch gebräuchliche histologische Vorbehandlung zuvor die übrigen Phosphat-Fraktionen weitgehend extrahiert wurden. Solche Präparate werden dann oft zu Unrecht als Nucleinsäure-Autoradiographien gedeutet.

Über die funktionelle Bedeutung der Phospho-Proteine ist bisher fast nichts bekannt. Das geschilderte Untersuchungsverfahren mag ein Ansatzpunkt sein, mit histochemischer Methodik das Wissen darüber zu erweitern.

15 J. H. T a ylor u. S. H. T a ylor, J. Heredity 44, 129 [1953]. 